Ann. Zootech., I979, 28 (I), I-r7.

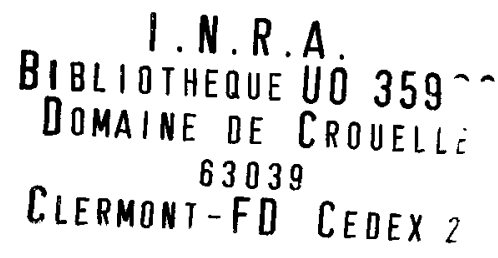

\title{
Utilisation des protéines par le veau préruminant à l'engrais VII. - Influence du remplacement des protéines du lait par celles du soja ou de la fèverole sur l'évacuation gastrique
}

\author{
P. GUILLOTEAU, R. TOULLEC, D. SAUVANT* et J.-L. PARUELLE** \\ avec la collaboration technique de Marguerite BraUriLs, \\ s. Boussion, J. LAREynie et Y. Manis \\ Station de Recherchcs Zootechniques, \\ Centre de Recherches de Rennes, I.N.R.A., \\ 65, we de Saint-Brieuc, 35072 Rennes Cedex (France) \\ * Chaire de Zootechnie, \\ Institut National Agronomique de Paris-Grignon, \\ I6, rue Claude-Bernard, 75005 Paris (France) \\ **U.C.A.A.B., B.P. 75, o2 foo Château-Thievry (France)
}

\begin{abstract}
Résumé
Les effets du remplacement du lait écrémé de l'aliment d'allaitement par des concentrés protéiques de soja ou de féverole, sur l'évacuation gastrique, ont été étudiés chez le veau préruminant. Le concentré de soja a été préparé en éliminant le saccharose et les $\alpha$ galactosides du tourteau par extraction éthanolique. Le concentré de féverole a été obtenu par extraction aqueuse à $\mathrm{pH} 8,5$. L'analyse factorielle des correspondances a été appliquée à l'ensemble des données. Pour chacun des 3 aliments, les variations individuelles sont importantes suivant la journée de mesures mais elles sont indépendantes du veau prélevé. Avec les deux aliments contenant les protéines végétales, l'évacuation gastrique des matières azotées ainsi que celle des matières grasses sont accélérées et les protéines sont moins hydrolysées, surtout dans le cas de la féverole : à l'issue des 3 heures qui suivent le repas, les quantités d'azote total et d'azote protéique parvenues dans le duodénum sont respectivement multipliées par I,2 et I,7 (soja) et 1,6 et 2,3 (féverole). La digestion des aliments dans la caillette est donc influencée par l'origine des protéines alimentaires. Les modifications observées en remplaçant les protéines du lait par celles du soja ou de la féverole pourraient être l'une des causes de la digestibilité moins élevée de ces dernières.
\end{abstract}

\section{Introduction}

Le remplacement du lait écrémé par d'autres sources de protéines dans les aliments d'allaitement distribués au veau préruminant peut entraîner des modifications importantes dans les phénomènes digestifs au niveau de la caillette et 
par voie de conséquence, au niveau intestinal. La plupart des travaux ont mis en évidence une accélération de l'évacuation gastrique des protéines et des matières grasses (Colvin, Lowe et Ramsey, i969; Toul, FrANTZEN et al., I973; GUILloteAu et al., I975). Cependant, cette accélération est variable suivant l'origine des protéines et les traitements technologiques qu'elles ont subis. Smith et Sissons (I975) ont au contraire observé une inhibition de l'évacuation gastrique avec certains tourteaux ou isolats de soja. Nous avons cherché à compléter ces observations en étudiant l'influence du remplacement des protéines du lait écrémé par celles de concentrés protéiques issus du soja ou de la féverole.

\section{TABIEAU I}

Composition des aliments

Composition of the diets

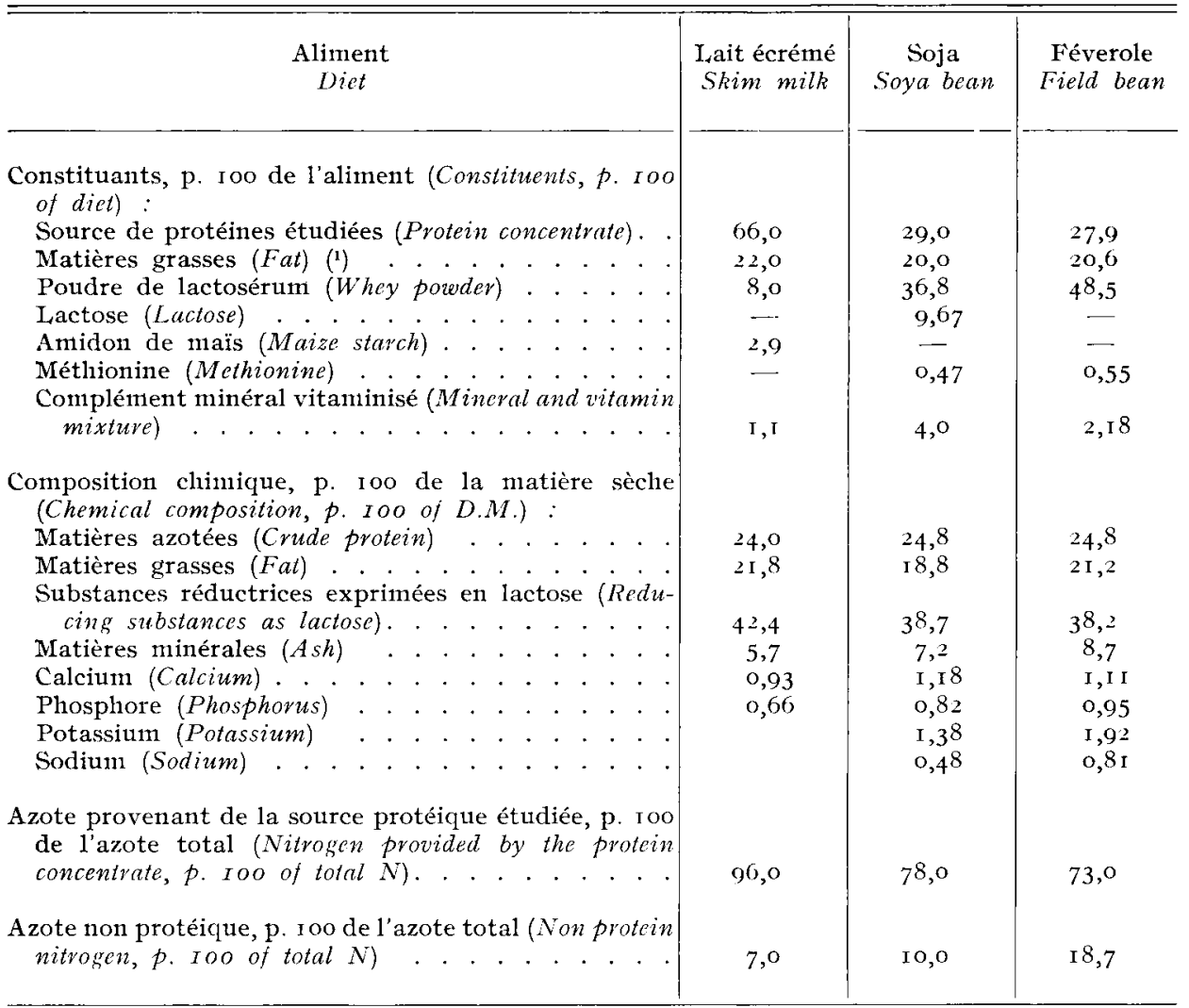

(1) I.es matières grasses avaient été incorporées à raison de 40 p. roo de la matière siche dans du lait écrémé concentré (aliment Lait écrémé) ou de 35 p. Ioo dans du lactosérum concentré (aliments Soja et Féverole); les 2 mélanges liquides ainsi obtenus avaient été homogénéisés et séchés par le procédé Spray. Fat was mixed in concentrated skim milk at the level of 40 p. Ioo of dry matter (skim milk diet) or in concentrated whey at the level of 35 p. 100 of dry matter (soya bean and field bean diets); these mixtures were homogenizcd assd spray dried. 


\section{Matériel et méthodes}

\section{Aliments}

Trois aliments d'allaitement, contenant près de $25 \mathrm{p}$. Ioo de matières ázotées par rapport à la matière sèche, ont été préparés (tabl. I). Dans l'aliment témoin (aliment Lait écrémé), les protéines provenaient presque exclusivement du lait écrémé. Dans les 2 autres aliments (aliments Soja et Féverole), la majeute partie ( $7^{8}$ ou 73 p. Ioo) des protéines était apportée par un concentré protéique de soja ou de féverole, le reste par du lactosérum et de la méthionine. Le concentré de soja contenait 59,6 p. Ioo de matières azotées par rapport à la matière sèche; il s'agissait d'un tourteau traité par la chaleur humide et dont le saccharose et les $\alpha$ galactosides avaient été éliminés par extraction à l'alcool (HAYpro-T', HAyEs Ltd, AsHOOD, ISRAEL). Le concentré de féverole contenait 65,0 p. Ioo de matières azotées; il avait été préparé à partir de graines décortiquées et broyées, par extraction aqueuse à $\mathrm{pH} 8,5$, élimination de la majeure partie des constituants insolubles par décantation et séchage par le procédé Spray (Laboratoire de Technologie Laitière, I.N.R.A., Rennes).

Les aliments d'allaitement ont été dilués au moment de l'emploi dans de l'eau à $40^{\circ} \mathrm{C}$, à raison de $125 \mathrm{~g}$ de matière sèche par $\mathrm{kg}$ de lait de remplacement. Les laits ainsi obtenus ont été distribués au seau 2 fois par jour, les quantités offertes variant selon le poids des veaux de 3,5 à $5 \mathrm{~kg}$ par repas.

\section{Animaux, prélèvements et analyses chimiques}

Sept veaux mâles (6 Frisons et I Normand) ont été munis d'une canule duodénale réentrante (AsH, I 962), la partie proximale étant placée ro $\mathrm{cm}$ environ après le pylore (tabl. 2). Les mesures ont commencé I5 jours après la pose des canules, l'aliment étudié étant distribué depuis au moins une semaine. Lors de chaque journée de prélèvement, les digesta sortant de la caillette ont été recueillis en totalité dans la glace fondante pendant 7 périodes réparties sur 7 heures consécutives après le repas du matin; ils ont été simultanément réintroduits dans le duodénum après prise d'échantillons représentatifs destinés aux analyses. Les autres détails concernant les méthodes de prélèvement et d'analyse chimique ont été rapportés précédemment (GUILLOTEAU et al., I975).

\section{Analyses statistiques}

Les courbes d'évacuation gastrique de la matière fraîche, de la matière sèche et de ses constituants ont été comparées à l'aide de la distance du $\mathrm{D}^{2}$ de MAHALANoBIs, décrite par RAO (I952).

Cette première approche a été complétée par plusieurs analyses factorielles des correspondances, de manière à :

- étudier l'ensemble des relations entre les facteurs de variation (aliment, animal, jour de prélèvement) susceptibles de modifier l'allure des cinétiques d'évacuation gastrique; 
TABLEAU 2

Animaux et prélèvements

Animals and abomasal digesta collection

\begin{tabular}{|c|c|c|c|c|c|c|}
\hline \multirow{2}{*}{$\begin{array}{l}N^{0} \text { du veau } \\
N^{0} \text { of calf }\end{array}$} & \multirow{2}{*}{$\begin{array}{c}\text { Age } \\
\text { au moment } \\
\text { de l'opération } \\
\text { (j) } \\
\text { Age } \\
\text { on operation } \\
(d)\end{array}$} & \multirow{2}{*}{$\begin{array}{c}\text { Poids vif } \\
\text { au moment } \\
\text { de l'opération } \\
\text { (kg) } \\
\text { Live weight } \\
\text { on operation } \\
(\text { kg })\end{array}$} & \multicolumn{3}{|c|}{$\begin{array}{l}\text { Nombre de jours de prélèvements } \\
\text { après ingestion des aliments } \\
\text { Number of days of digesta collection with }\end{array}$} & \multirow{2}{*}{$\begin{array}{c}\text { Nombre } \\
\text { de jours de } \\
\text { prélèvements } \\
\text { par veau } \\
\text { Number } \\
\text { of days of } \\
\text { digesta } \\
\text { collection } \\
\text { per calf }\end{array}$} \\
\hline & & & $\begin{array}{l}\text { Lait écrémé } \\
\text { Skim milk }\end{array}$ & $\begin{array}{c}\text { Soja } \\
\text { Soya bean }\end{array}$ & $\begin{array}{l}\text { Féverole } \\
\text { Ficld bean }\end{array}$ & \\
\hline 688. & I 6 & $5^{\circ}$ & I & - & - & I \\
\hline $69 \mathrm{I}$. & 22 & 50 & 2 & 一 & I & 3 \\
\hline 598 . & 23 & 52 & - & 3 & I & 4 \\
\hline 599 . & I 7 & $5 \mathrm{I}$ & I & - & - & I \\
\hline 604. & I 7 & $5^{\circ}$ & - & 2 & 2 & 4 \\
\hline 644. & 22 & 48 & - & I & - & I \\
\hline 646. & 22 & 57 & 2 & - & 2 & 4 \\
\hline \multicolumn{3}{|c|}{$\begin{array}{l}\text { Nombre total de prélèvements avec } \\
\text { chaque régime (Total number of days } \\
\text { of digesta collection with each diet }) .\end{array}$} & 6 & 6 & 6 & \\
\hline
\end{tabular}

- apprécier les valeurs respectives des différents modes d'expression (quantités recueillies par périodes ou quantités cumulées) utilisables pour comparer les régimes; gastrique;

- rechercher 1'existence de phases différentes dans la cinétique d'évacuation

- estimer l'importance des variations individuelles.

Cette méthode d'analyse statistique descriptive, mise au point par BENZECRI (I973), permet de mettre en évidence les principales causes de variations des courbes d'évacuation gastrique par rapport à une évolution moyenne. L'analyse factorielle des correspondances s'interprète graphiquement par la projection simultanée des 7 points de temps de prélèvement et des I44 courbes cinétiques sur le plan qui traduit les principales variations des données. Sur ce plan, les proximités entre les courbes cinétiques sont proportionnelles à leur ressemblance. Le même mode de raisonnement s'applique pour les points correspondant aux temps de prélèvement. En outre, une courbe cinétique présente une valeur d'autant plus importante à une période donnée de prélèvement que les deux points correspondants sont proches.

\section{Résultats}

Les variations individuelles et journalières dans les débits de la matière fraîche, de la matière sèche et de ses constituants sont importantes, surtout pendant les deux premières heures après le repas, comme l'indiquent les valeurs des écarts types (tab1. 3). Ce phénomène est observé avec les trois aliments et sera développé dans le cadre de l'analyse multivariate. 


\section{TABLEAU 3}

Évacuation gastrique de la matière sèche et de ses constituants : quantités émises par demi-heure en p. Ioo des quantités ingérées (moyennes et écarts types de l'échantillon)

Abomasal emptying of dry matter and of its constituents : collected amounts per $30 \mathrm{mn}$ in p. Ioo of ingested amounts (means and standard-errors)

\begin{tabular}{|c|c|c|c|c|c|c|c|}
\hline $\begin{array}{r}\text { Te } \\
\text { après le } \\
T \\
\text { after }\end{array}$ & $\begin{array}{l}\text { emps } \\
\text { e repas }(\mathrm{h}) \\
\text { ime } \\
\text { meal (h) }\end{array}$ & $\begin{array}{c}\text { Aliment } \\
\text { Diet }\end{array}$ & $\begin{array}{c}\text { Matière } \\
\text { sèche } \\
\text { Dry } \\
\text { matter }\end{array}$ & $\begin{array}{c}\text { Matières } \\
\text { minérales } \\
A s h\end{array}$ & $\begin{array}{c}\text { Substances } \\
\text { réductrices } \\
\text { Reducing } \\
\text { substances }\end{array}$ & $\begin{array}{c}\text { Matières } \\
\text { grasses } \\
\text { Fat }\end{array}$ & $\begin{array}{l}\text { Matières } \\
\text { azotées } \\
\text { Crude } \\
\text { protein }\end{array}$ \\
\hline $0-0,5$ & . & $\begin{array}{l}1 \\
2 \\
3\end{array}$ & $\begin{array}{ll}\text { I } 7,0 & (6,4) \\
2 \mathrm{I}, 3 & (7,9) \\
26,7 & (\mathbf{I} I, 3)\end{array}$ & $\begin{array}{ll}27,7 & (12,9) \\
21,3 & (7,2) \\
27,8 & (13,2)\end{array}$ & $\begin{array}{ll}23,0 & (\mathrm{I} I, 4) \\
22,2 & (9,6) \\
24,3 & (\mathrm{I} 0,5)\end{array}$ & $\begin{array}{rr}5,9 & (5,7) \\
18,9 & (6,1) \\
22,7 & (7,0)\end{array}$ & $\begin{array}{ll}\text { I } 2,3 & (7,8) \\
\text { I } 4,9 & (6,4) \\
26,6 & (\text { I } 3,4)\end{array}$ \\
\hline $0,5^{-I, O}$ & $\cdot$ & $\begin{array}{l}\text { I } \\
2 \\
3\end{array}$ & $\begin{array}{l}9,0 \quad(9,7) \\
6,6 \quad(6,3) \\
3,0 \quad(3,2)\end{array}$ & $\begin{aligned} \text { I } 4,2 & (\text { I } 4,4) \\
7,3 & (6,9) \\
3, \mathrm{I} & (3,2)\end{aligned}$ & $\begin{array}{ll}8,4 & (10,4) \\
8,5 & (8,6) \\
2,7 & (3, I)\end{array}$ & $\begin{array}{ll}2,3 & (2,7) \\
4,7 & (3,3) \\
2,6 & (3,1)\end{array}$ & $\begin{array}{ll}5,3 & (5,9) \\
3,7 & (3,2) \\
2,9 & (2,8)\end{array}$ \\
\hline $\mathrm{I}, \mathrm{O}-\mathrm{I}, \mathbf{5}$ & . . & $\begin{array}{l}\text { I } \\
2 \\
3\end{array}$ & $\begin{array}{ll}8,8 & (6,7) \\
7,2 & (4,0) \\
9,0 & (4,5)\end{array}$ & $\begin{array}{rr}3,6 & (9,3) \\
8,2 & (4,4) \\
\text { I } 0,1 & (4,8)\end{array}$ & $\begin{array}{rr}\text { I } 2,2 & (9,4) \\
9, \text { I } & (5,3) \\
8,6 & (4,2)\end{array}$ & $\begin{array}{ll}2, I & (2,1) \\
6,7 & (3,7) \\
8,0 & (4,4)\end{array}$ & $\begin{array}{ll}5,7 & (4,1) \\
3,6 & (2,2) \\
8,6 & (3,9)\end{array}$ \\
\hline $\mathrm{I}, 5^{-2}, \mathrm{O}$ & . & $\begin{array}{l}\mathbf{I} \\
2 \\
3\end{array}$ & $\begin{array}{ll}3,2 & (2,7) \\
7,4 & (10,8) \\
4,7 & (4,4)\end{array}$ & $\begin{array}{ll}5,2 & (4,4) \\
8,7 & (13, I) \\
5,0 & (4,6)\end{array}$ & $\begin{array}{ll}4,8 & (4,0) \\
8,4 & (\text { I I }, 4) \\
4,4 & (3,5)\end{array}$ & $\begin{array}{ll}1,2 & (0,8) \\
7,2 & (8,9) \\
4,3 & (4,7)\end{array}$ & $\begin{array}{ll}2,2 & (1,6) \\
8,4 & (\mathrm{I} I, 3) \\
4,6 & (4,8)\end{array}$ \\
\hline $2,0-3,0$ & . . & $\begin{array}{l}\mathbf{I} \\
2 \\
3\end{array}$ & $\begin{array}{ll}5,2 & (1,2) \\
6,2 & (1,7) \\
5,8 & (3,6)\end{array}$ & $\begin{array}{ll}7,9 & (1,8) \\
6,7 & (1,4) \\
6,0 & (3,3)\end{array}$ & $\begin{array}{ll}6,0 & (2,4) \\
6,3 & (1,3) \\
5,6 & (3,6)\end{array}$ & $\begin{array}{ll}4,6 & (3,6) \\
5, I & (1,2) \\
3,8 & (2,8)\end{array}$ & $\begin{array}{ll}3,9 & (1,2) \\
5,2 & (2,3) \\
4,6 & (3,3)\end{array}$ \\
\hline $3,0-5,0$ & . $\cdot$ & $\begin{array}{l}1 \\
2 \\
3\end{array}$ & $\begin{array}{ll}4,6 & (\mathrm{I}, 7) \\
4,8 & (\mathrm{o}, 6) \\
3,4 & (\mathrm{I}, 4)\end{array}$ & $\begin{array}{ll}6,0 & (2,2) \\
5,7 & (0,8) \\
3,7 & (\mathrm{I}, 5)\end{array}$ & $\begin{array}{ll}3,4 & (2,8) \\
3,8 & (1,1) \\
3,2 & (1,7)\end{array}$ & $\begin{array}{ll}4,3 & (\mathrm{I}, 7) \\
4,4 & (0,5) \\
3,0 & (\mathrm{I}, \mathrm{O})\end{array}$ & $\begin{array}{ll}4,6 & (\mathrm{I}, 2) \\
4,3 & (\mathrm{I}, 5) \\
3,4 & (\mathrm{I}, 4)\end{array}$ \\
\hline $5,0-7,0$ & $\cdot$ & $\begin{array}{l}\text { I } \\
2 \\
3\end{array}$ & $\begin{array}{ll}3,0 & (0,7) \\
3,6 & (1,7) \\
3,7 & (2,3)\end{array}$ & $\begin{array}{ll}3,5 & (0,7) \\
3,9 & (0,8) \\
4,3 & (3,1)\end{array}$ & $\begin{array}{ll}2,2 & (3,3) \\
0,9 & (0,7) \\
4,9 & (5,5)\end{array}$ & $\begin{array}{ll}4, \mathrm{I} & (\mathrm{I}, 3) \\
2,2 & (0,6) \\
3,5 & (2,2)\end{array}$ & $\begin{aligned} 4,0 & (1,2) \\
\text { I I , I } & (12,4) \\
4,0 & (2,7)\end{aligned}$ \\
\hline
\end{tabular}

1. Aliment Lait écrémé (Skim milk diet); 2. Aliment Soja (Soya bean diet); 3. Aliment Féverole (Field bean diet). 


\section{Évacuation gastrique de la matière fraîche et de la matière sèche}

Excepté pendant la première demi-heure qui suit le repas, le débit de la matière fraîche est plus faible avec l'aliment Féverole qu'avec les deux autres aliments. Pour l'ensemble des 7 heures de mesure, la courbe cumulée d'évacuation gastrique (fig. I) obtenue lorsque les veaux reçoivent l'aliment Féverole se distingue significativement des deux autres. L'examen de ces courbes indique qu'il faut environ $4 \mathrm{~h}, 4 \mathrm{~h}$ I $5 \mathrm{mn}$ et $6 \mathrm{~h}$ pour obtenir une quantité équivalente à la quantité ingérée lorsque les animaux reçoivent respectivement les aliments Lait écrémé, Soja et

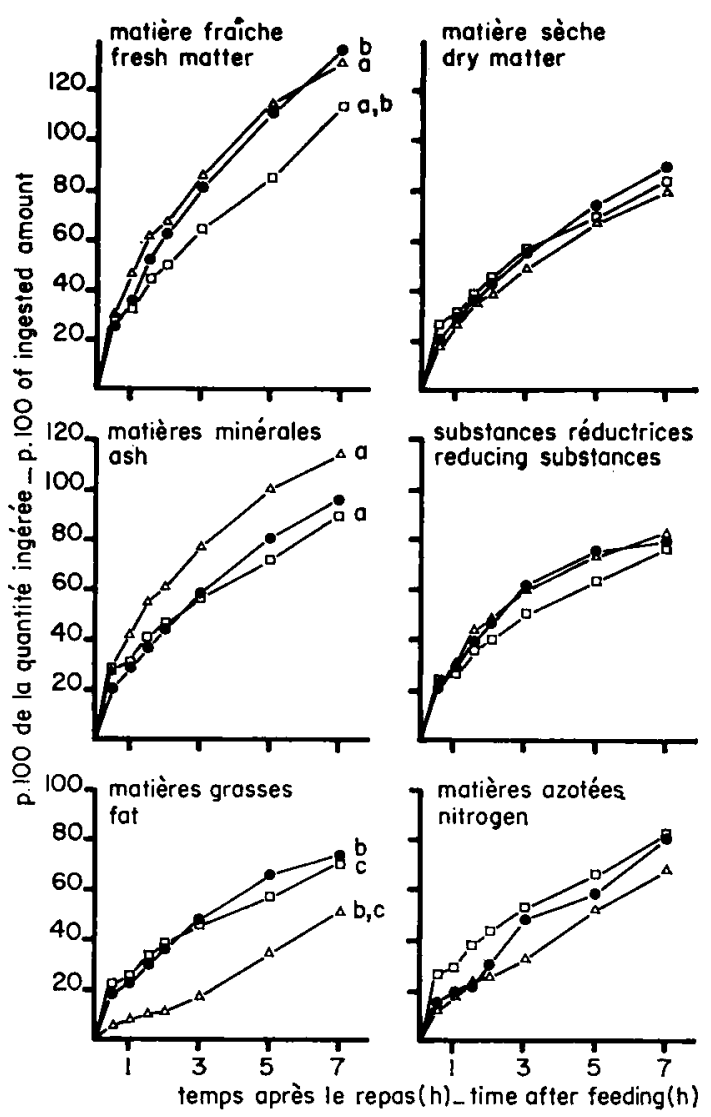

FIG. I. - Évacuation gastrique de la matière frâ̂che, de la matière sèche et de ses constituants (quantités cumulées en p. Ioo des quantités ingérées).

Les courbes accompagnées de la même lettre sont significativement différentes $\left(P<0,0_{5}\right)$.

Abomasal emptying of fresh matter, dry matter and of its constituents (cumulative amounts in p. Ioo of ingested ones).

The curves with the same letter are significantly different $(P<0.05)$.

$\Delta \longrightarrow \Delta$ Aliment lait écrémé (Skim milk diet)

-_- Aliment soja (Soya bean diet)

Aliment féverole (Field bean diet) 
Féverole. Les quantités totales obtenues en $7 \mathrm{~h}$ sont respectivement de I32, I3I et II5 p. Ioo par rapport aux quantités ingérées.

Au cours de la première demi-heure après le repas, l'évacuation gastrique de la matière sèche est plus rapide avec les aliments Soja et surtout Féverole qu'avec l'aliment Lait écrémé : les quantités totales recueillies atteignent respectivement $2 \mathrm{I}, 27$ et I7 p. Ioo des quantités ingérées (tabl. 3). Par la suite les débits sont plus faibles avec l'aliment Féverole qu'avec les deux autres aliments. Toutefois, aucune différence significative n'est observée entre les 3 courbes cumulées (fig. I).

\section{Évacuation gastrique des constituants de la matière sèche}

Avec l'aliment Lait écrémé, les substances réductrices et les matières minérales quittent la caillette plus rapidement que les matières azotées et surtout que les lipides : les quantités totales récoltées au cours des 2 premières heures après le repas atteignent respectivement $48,6 \mathrm{I}, 25$ et II $\mathrm{p}$. Ioo des quantités ingérées (tab1. 3). Les différences observées sont significatives sur l'ensemble des 7 heures de mesure. En revanche, avec l'aliment Féverole, tous les constituants de la matière sèche quittent la caillette sensiblement à la même vitesse : les quantités totales collectées au cours des deux premières heures après le repas sont respectivement de $40,46,43$ et $38 \mathrm{p}$. roo. Avec l'aliment Soja, les résultats ont tendance à être intermédiaires entre ceux obtenus avec les deux autres aliments : les quantités totales recueillies pendant le même temps sont respectivement de $48,46,3$ I et 37 p. Iоo; les courbes cumulées d'évacuation gastrique des substances réductrices et des matières azotées sont significativement différentes. Les quantités totales de matières minérales recueillies en 7 heures sont supérieures aux quantités ingérées lorsque les veaux reçoivent l'aliment Laait écrémé (I I 4 p. Ioo); elles leursont pratiquement égales av ec l'aliment Soja (97 p. I00) et inférieures avec l'aliment Féverole (90 p. IOO).

L'évacuation gastrique des matières azotées et surtout celle des matières grasses sont plus rapides pour les deux aliments expérimentaux que pour l'aliment Témoin (fig. I). Ainsi, les quantités totales de matières azotées émises au cours des 3 premières heures qui suivent le repas sont multipliées respectivement par $I, 2$ et $r, 6$. Toutefois, seules les courbes cumulées d'évacuation gastrique des matières grasses des veaux recevant les aliments Soja et Féverole diffèrent significativement de celle des veaux ingérant l'aliment Lait écrémé.

\section{Azote non protéique et azote protéique}

Quand les veaux reçoivent l'aliment Lait écrémé, la proportion d'azote non protéique (soluble dans l'acide trichloracétique I2 p. Ioo) est beaucoup plus élevée dans les digesta que dans l'aliment (49 p. Ioo de l'azote total au lieu de 7 ), aussitôt après le repas (fig. 2); elle reste constante pendant 3 heures puis diminue jusqu'à 3I p. Ioo. Avec l'aliment Soja, la proportion dans les digesta est également supérieure à celle observée dans l'aliment ( $26 \mathrm{p}$. xoo au lieu de ro) pendant les 30 premières minutes; par la suite, elle varie de manière irrégulière entre $3 \mathrm{I}$ et $43 \mathrm{p}$. Ioo. En revanche, avec l'aliment Féverole, la proportion dans les digesta est d'abord voisine de celle trouvée dans l'aliment (22 p. Ioo au lieu de 17 ) puis elle augmente régulièrement jusqu'à $54 \mathrm{p}$. roo. 


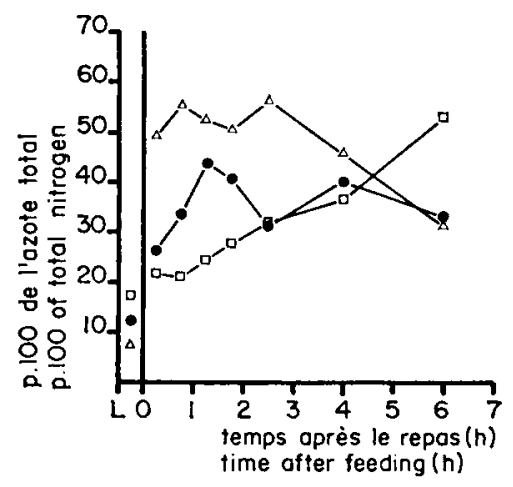

FIG. 2. - Evolution du taux d'azote non protéique dans les digesta ( $p$. Ioo de l'azote total). Evolution of non protein nitrogen level in digesta ( $p$. Ioo of total nitrogen).

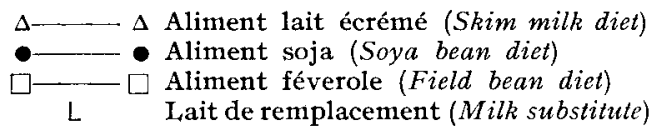

En négligeant les matières azotées endogènes sécrétées en amont du pylore on peut estimer qu'au minimum environ 25 , I6 et 8 p. Ioo de l'azote protéique ingéré seraient transformés en azote non protéique dans la caillette au cours des $7 \mathrm{~h}$ qui suivent le repas quand les veaux reçoivent respectivement les aliments Lait écrémé, Soja et Féverole. Les quantités d'azote protéique parvenant dans le duodénum sont plus importantes avec les aliments Soja et Féverole puisque à l'issue des 3 h qui suivent le repas, elles sont respectivement I,7 et 2,3 fois plus élevées (fig. 3); cependant, les courbes cumulées ne sont pas significativement différentes.

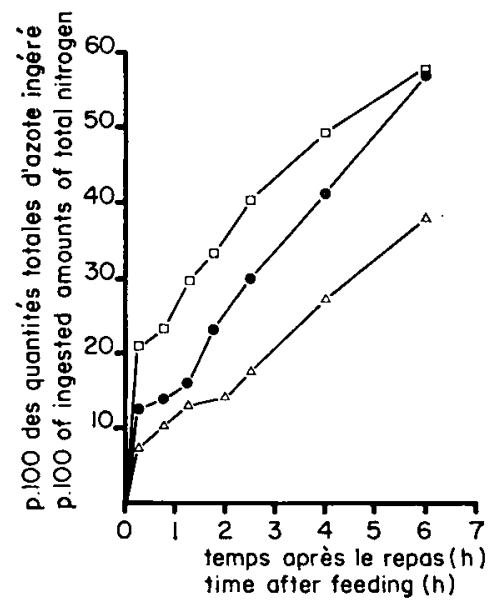

Fig. 3. - Évacuation gastrique de l'azote protéique (quantités cumulées en p. Ioo des quantités d'azote total ingéré).

Abomasal emptying of protein nitrogen (cumulative amounts in p. Ioo of ingested amounts of toial nitrogen).

$\Delta-\Delta$ Aliment lait écrémé (Skim milk diet)

- Aliment soja (Soya bean diet)

$\square$ Aliment févérole (Field bean diet) 


\section{Évolution $d u p H$}

Le $\mathrm{pH}$ des digesta est très acide immédiatement avant le repas ( 1,6 à 2,3$)$; il augmente brutalement au cours des cinq minutes suivantes pour atteindre $5, \mathrm{I}$ à 6,3 (fig. 4). Pendant les 7 heures qui suivent le repas, le $\mathrm{pH}$ diminue régulièrement avec tous les aliments, surtout avec les aliments expérimentaux; toutefois il demeure toujours plus élevé avec l'aliment Féverole.
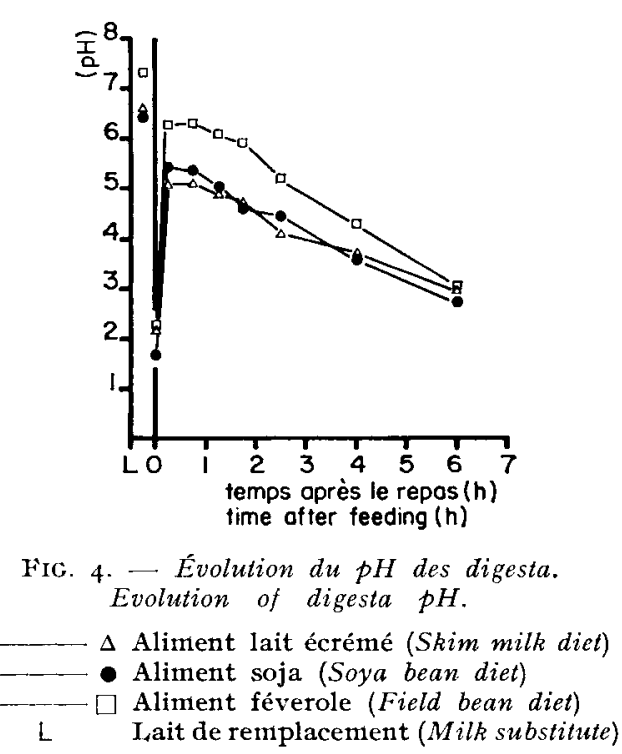

\section{Analyse multivariate}

L'analyse factorielle des correspondances prend en compte les I 44 cinétiques d'évacuation gastrique (quantités cumulées en p. Ioo des quantités ingérées). Elle permet de retenir deux facteurs qui expliquent 76,4 p. Ioo de la variation globale. Il est donc possible de représenter par projection orthogonale des otservations et des caractères sur le plan défini par ces axes principaux (facteur I et facteur 2) la majeure partie de l'information intéressante de cette expérience (fig. 5). Le premier axe est prédominant puisqu'il représente $64,0 \mathrm{p}$. Ioo de la dispersion des données. Sur ce plan de meilleure description de celle-ci, il est possible de tracer deux nouveaux axes ( $12 \mathrm{a}$ et $\mathrm{r} 2 \mathrm{~b}$ ) qui permettent de mieux interpréter biologiquement les résultats (BENZECRI, I973).

L'axe raa permet de définir deux phases bien distinctes dans l'évacuation gastrique : la première période (temps 0,5 ) se distingue de l'ensemble des 6 autres. Pendant cette première phase, les digesta en provenance de l'aliment Féverole ont une évacuation plus rapide que ceux issus des deux autres aliments puisque tous ses constituants sont plus proches du point 0,5 . L'axe $\mathrm{I} 2 \mathrm{~b}$ indique la vitesse relative de sortie des constituants des digesta en fonction du temps après le repas, première demi-heure exclue. Les constituants quittant la caillette le plus lentement 


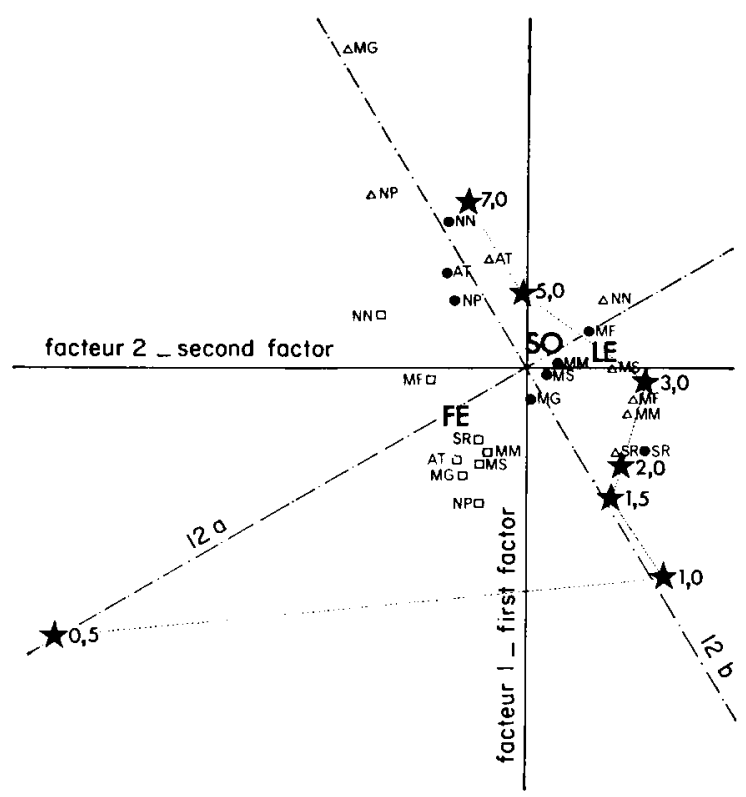

HIG. 5. - Evacuation gastrique de la matière fraîche, de la matière sècne et de ses constituants (quantités cumulées en p. Ioo des quantités ingérées) : projection des observations et des caractères sur le plan principal I-2 de l'analyse factorielle des correspondances.

Abomasal emptying of tresh matter, dry matter and its constituents (cumulative amounts in p. 100 of ingested amounts) : projection of observations and characters on the main plan $I-2$ of correspondance analysis.

$\star \quad$ Temps, h (Time, $h$ ).

LE Aliment Lait écrémé (Skim milk diet)

SO Aliment Soja (Soya bean diet)

FE Aliment Féverole (Field bean diet)

$\Delta \quad$ Aliment Lait écrémé (Skim milk diet)

- Aliment Soja (Soya bean diet)

$\square \quad$ Aliment Féverole (Field bean diet)

Point mioyen pour l'ensemble de l'expérience

MF Matière fraîche (Fresh matter).

MS Matière sèche (Dry matter).

MM Matières minérales $(A s h)$.

SR Substances réductrices (Reducing substances).

MG Matières grasses (Fat).

AT Azote total (Total nitrogen).

NP Azote protéique (Protein nitrogen).

NN Azote non protéique (Non protein nitrogen).

par exemple les matières grasses (MG) et les matières azotées totales (AT) de l'aliment Lait écrémé, se situent du côté des points 5,0 et $7,0 \mathrm{~h}$ après le repas.

D'une façon générale, nous retrouvons les observations effectuées à partir de l'examen des figures $I$ et 3 . C'est avec l'aliment Lait écrémé que la dispersion des points représentant les constituants des digesta est la plus forte et c'est avec l'aliment Féverole qu'elle est la plus faible. La plus grande différence entre les trois aliments réside dans l'évacuation gastrique des matières azotées protéiques $(\mathrm{NP})$ et surtout dans celle des matières grasses (MG). En effet, les trois points $\mathrm{NP}$ sont épars sur la figure 5 suivant l'axe $\mathrm{I} 2 \mathrm{~b}$; les deux points MG correspondant aux aliments expérimentaux sont voisins entre eux, mais très éloignés de celui qui se rapporte à l'aliment témoin. 


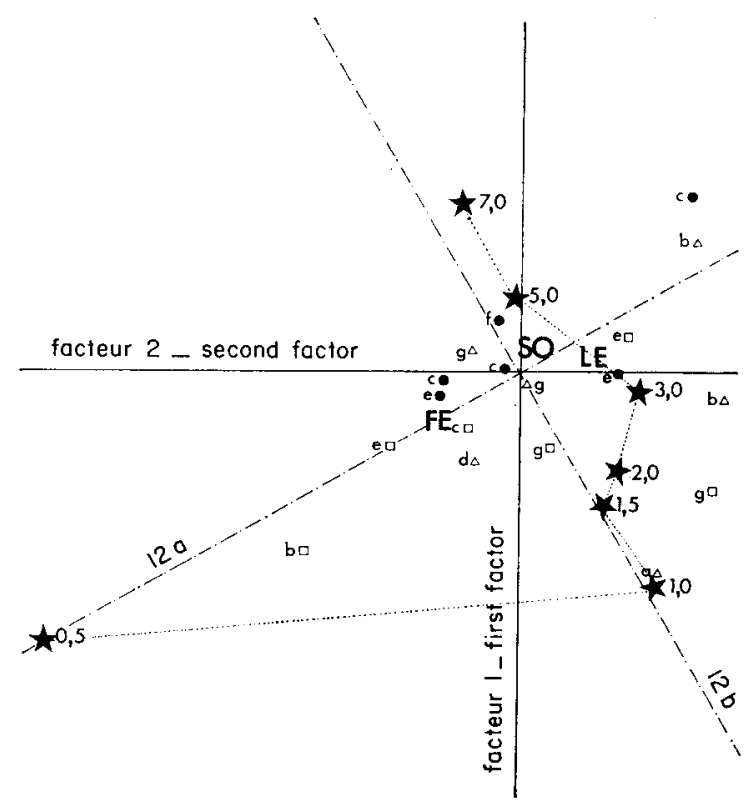

FIG. 6. - Évacuation gastrique à la suite de l'ingestion des aliments Lait écrémé, Soja et Féverole : projection des observations et des caractères sur le plan $1-2$ de l'analyse factorielle des correspondances. Chaque point représente l'ensemble des constituants, matières grasses et azote protéique exclus (quantités cumulées en p. Ioo des quantités ingérées) pour une journée de mesures.

Abomasal emptying after ingestion of Skim milk, Soya bean and Field bean diet : projection of obsevvations and characters on the main plan $T-2$ of correspondance analysis. Each point represents all the constituents without fat and protein nitrogen (cumulative amounts in $p$. Ioo of ingested amounts) for each day of digesta collection.

$\star \quad$ Temps, h (Time, $h$ ).

LE Aliment Lait écrémé (Skim milk diet)

SO Aliment Soja (Soya bean diet)

FE Aliment Féverole (Field bean diet)

Point moyen pour l'ensemble de l'expérience (matières grasses et azote protéique exclus) (Mean point for the whole experiment (without fat and protein nitrogen)). $\Delta \quad \begin{array}{ll}\text { Aliment Lait écrémé (Skim milk diet) } \\ \text { Aliment Soja (Soya bean diet) }\end{array} \quad \begin{gathered}\text { Point moyen par journée de mesure (matières } \\ \text { grasses et azote protéique exclus) (Mean }\end{gathered}$

Point moyen par journée de mesure (matières Aliment Féverole (Field bean diet) point for each day of digesta collection (without fat and protein nitrogen).
Veau no 688 (Calf $n^{\circ} 688$ ).
Veau no 69I (Calf $n^{\circ} 69 I$ ).
Veau $\mathrm{n}^{\mathrm{o}} 598$ (Calf $n^{\circ} 598$ ).
Veau no 599 (Calf $n^{\circ} 599$ ).
Veau no 604 (Calf $n^{\circ}$ 604).
Veau no 644 (Calf no 644).
Veau no 646 (Calf $n^{\circ} 646$ ).

De plus, des aspects nouveaux sont mis en évidence. Les matières azotées non protéiques (NN) sortent plus lentement que les substances réductrices (SR), les matières minérales (MM), la matière sèche (MS) et la matière fraîche (MF), quel que soit l'aliment ingéré. Eilles sortent plus lentement que les matières azotées protéiques (NP) et les matières azotées totales dans le cas du soja et surtout de la féverole et plus rapidement avec l'aliment Lait écrémé.

La projection sur le plan des facteurs I et 2 des points moyens représentant chacun une journée de prélèvement (fig. 6) fait apparaître un éclatement princi- 


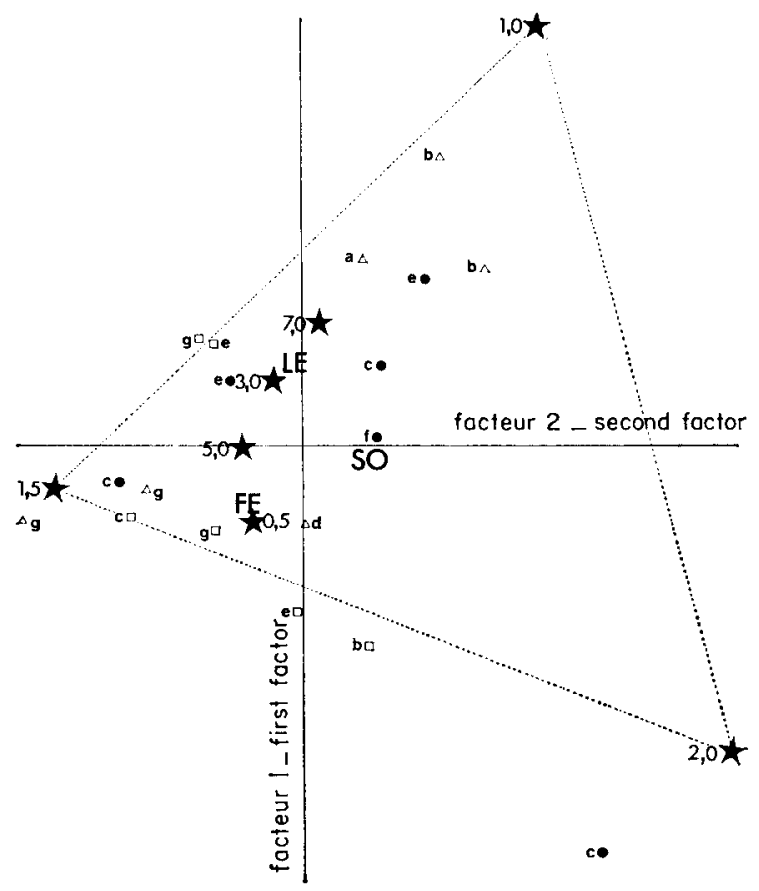

FIG. 7. - Évacuation gastrique à la suite de l’ingestion des aliments Lait écrémé, Soja et Féverole : projection des observations et des caractères sur le plan principal $I-2$ de l'analyse factorielle des correspondances. Chaque point représente l'ensemble des constituants (quantités récoltées par période en p. Ioo des quantités ingérées) pour une journée de mesures.

L'examen de cette figure montre qu'il y a un éclatement des journées de mesure indépendamment du veau prélevé. Ceci serait en velation avec les périodes de prélèvements après le vepas et plus particulièrement la deuxième (temps $x, o$ ), la troisième (temps $I, 5$ ) et la quatrième (temps 2,0$)$. Par exemple, la proximité d’une des journées de mesure effectuées chez le veau 598 quand il a reçu l'aliment Soja et de la quatrième période (temps 2,0 ) indique qu'il y avait un débit important pendant celle-ci; en revanche, très peu de digesta ont été recueillis pendant la deuxième (temps $I, 0)$ et la troisième (temps $I, 5$ ) périodes. La description en triangle des points $I, O-I, 5$ et 2,0 montre qu'il y a un effet compensatoire important entre les résultats des trois périodes coryespondantes.

Abomasal emptying after ingestion of Shim milk, Soyabean and Field bean diets : projection of observations and characters on the main plan $I-2$ of correspondance analysis. Each point represents all the constituents (cumulative amounts per period in $p$. 1 oo of ingested amounts) for each day of digesta collection.

The figuve shows that there is a dispersion of the collection days independently of the collected calf. This seems to be in relation with the sampling period after feeding and more particularly the second (time I.o), the third (time I.5) and the fourth (time 2.0). For example, the proximity of one of the collection days made on the calf 598 when fed the Soya bean diet and of the fourth period (time 2.o) indicates that there was a lavge out flow during this one; on the contrary, very little digesta was collected for the second (time I.o) and the third (time I.5) periods. The triangular description of points I.O-I.5 and 2.O shows that there is an important compensatory effect between the results of the three corresponding periods.

Temps, h (Time, h).

Aliment Lait écrémé (Skim milh diet) \ Point moyen pour l'ensemble de l'expérience Aliment Soja (Soya bean diet) $\quad$ (Mean point for the whole experiment).

Veau no 688 (Calf $n^{\circ}$ 688).

Point moyen par journée de mesures (Mean point for each day of digesta collection). 
palement suivant 1'axe I2a. La position du point 0,5 montre que les différences entre journées de prélèvement se manifestent plus particulièrement au cours de la première demi-heure de prélèvement. $L_{1}$ e codage des points moyens suivant le régime alimentaire $(\Delta, \bullet, \square$ ) et l'animal (a à g) permet de constater qu'il n'y a pas de variations individuelles répétables. Le regroupement des projections orthogonales des périodes $\mathrm{I}, \mathrm{o}$ à 7,0 sur l'axe I2a montre qu'à partir de la seconde phase de prélèvement des phénomènes de compensation réduisent les différences entre les expérimentations. Cet effet compensatoire est le plus important entre les résultats des trois prélèvements correspondant aux périodes I,O-I,5 et 2,0 (fig. 7).

\section{Discussion}

Lorsque les veaux reçoivent l'aliment Lait écrémé, l'allure générale de 1'évacuation gastrique est semblable à celle qui a déjà été observée à la suite de l'ingestion du lait entier (MylReA, I966a et $b$; MATHLEU, I968; Toul, Ec, ThIVEND et MATHIEU, I97I; LEIBHOLZ, I975); en particulier, les substances réductrices et les matières minérales quittent la caillette plus rapidement que les matières azotées et les lipides. En revanche, quand les animaux consomment l'aliment Féverole, ces 4 constituants parviennent dans le duodénum sensiblement à la même vitesse; cela est dû̀ à l'absence de coagulation des protéines dans la caillette (ToulLE, Thivend, Mathieu, i97 I; Frantzen et al., I973; Guilioteau et al., i975). Les résultats intermédiaires obtenus avec l'aliment Soja pourraient être liés à des phénomènes de sédimentation, de précipitation ou de floculation, plus importants avec cet aliment qu'avec l'aliment Féverole.

Avec les 3 aliments, les quantités totales de matière fraîche recueillies pendant les $7 \mathrm{~h}$ qui suivent le repas sont supérieures aux quantités ingérées. Les quantités minimales de salive et de suc gastrique sécrétées pendant cette période peuvent être estimées à environ 2070,2280 et I $070 \mathrm{~g} / \mathrm{l} 00 \mathrm{~kg}$ de poids vif, respectivement avec les aliments Lait écrémé, Soja et Féverole. Le veau préruminant produirait environ $2.55 \mathrm{ml}$ de salive/ $100 \mathrm{~kg}$ de poids vif / $\mathrm{h}$ (SMITH, I959) et I, 51 de suc gastrique $/ 100 \mathrm{~kg}$ de poids vif $/ \mathbf{I} 2 \mathrm{~h}$ (Roy et SToвo, I975); la somme rámenée à $7 \mathrm{~h}$ serait de $2660 \mathrm{ml}$, ce qui n'est pas en contradiction avec les estimations que nous avons faites pour les 2 premiers aliments, car la sécrétion du suc gastrique dépend de la quantité de lait ingéré (A.SH, I964) et de l'âge de l'animal (Gurlooteau et I.E CALVE, I977). Dans notre essai, les quantités sécrétées pour l'aliment témoin sont probablement sous estimées du fait que les quantités totales de matière sèche recueillies en $7 \mathrm{~h}$ sont moins élevées qu'avec les aliments expérimentaux. WiLLIAMS, Roy et GILIIES (I976) ont observé une diminution dans la sécrétion de suc gastrique en remplaçant les protéines du lait par celles d'un tourteau de soja et une augmentation en utilisant des protéines de poisson. Les sécrétions de matières minérales sont plus faibles avec l'aliment Soja et surtout avec l'aliment Féverole, contrairement aux observations effectuées par Toul $\mathrm{L}_{\mathrm{EC}}$, Thivend et Mathieu

\footnotetext{
Veau $n^{\circ} 69 \mathrm{I}$ (Calf $n^{\circ} 69 I$ ).

Veau $n^{\circ} 598$ (Calf $n^{\circ} 598$ ).

Veau $n^{\circ} 599$ (Calf $n^{\circ} 599$ ).

Veau no 604 (Calf no 604).

Veau $n^{\circ} 644$ (Calf $n^{\circ} 644$ ).

Veau $n^{\circ} 646$ (Calf no 646).
} 
(I97I) avec des protéines de lactosérum. Les sécrétions nettes d'eau et de minéraux par la muqueuse de la caillette et/ou par les glandes salivaires dépendent donc de l'origine des protéines alimentaires.

L'accélération observée dans l'évacuation gastrique des matières azotécs et des matières grasses avec les aliments expérimentaux a déjà été signalée avec des aliments dont les protéines proveraient du soja (CoLvin, LowE et RaMSEy, ig69; Ternouth et al., I975), de la féverole (Sissons et SMith, I975), du lactosérum ('Touldec, 'Thivend et Mathieu, I97I) ou du poisson ('Ternouth et al., I975; GUILLOTEAU et al., I975). Fille est principalement due à l'absence de coagulation des protéines mais elle est influencée par d'autres facteurs puisqu'elle varie suivant l'origine des protéines et les traitements technologiques qu'elles ont subis. Smith et Sissons (r975) ont même constaté une inhibition de 1'évacuation gastrique de la matière fraîche et des matières azotées avec certains produits issus du soja (tourteaux cuits ayant ou non subi une extraction aqueuse, isolats); en revanche, avec des tourteaux cuits traités par ébullition ou extraction dans l'éthanol aqueux, comme le concentré protéique de soja que nous avons utilisé, aucun ralentissement n'était observé; l'inhibition semblait liée à l'apparition de phénomènes d'allergie.

Dans notre essai, 1'évacuation gastrique de 1'aliment Féverole est plus rapide que celle de l'aliment Soja. L'aliment Féverole est plus riche en matières minérales, notamment en potassium et en sodium (tabl. I), ce qui pourrait modifier sa vitesse d'évacuation gastrique (BELL, et RAZIG, I973a et $b$ ); l'in fluence réelle de ce facteur dans un milieu aussi complexe est cependant difficile à apprécier à partir de ces données bibliographiques qui ont été obtenues à l'aide de solutions simples. Des différences de propriétés organoleptiques pourraient également intervenir, 1'aliment Féverole étant consommé plus lentement que les 2 autres aliments et l'évacuation gastrique étant en partie déterminée chez le veau par des influences d'origine encéphalique (BELL, Holbrooke et Titchen, I977).

Les protéines de l'aliment Soja et surtout celles de l'aliment Féverole sont moins hydrolysées dans la caillette que celles de l'aliment témoin. Cela peut être dû à leur temps de séjour plus court. Il est possible également que les enzymes protéolytiques de la caillette soient moins actives à l'égard des protéines du soja et de la féverole; ce phénomène pourrait être accentué par la valeur plus élevée du $\mathrm{pH}$, principalement avec 1'aliment Féverole, les valeurs optimales pour l'activité protéolytique générale de la chymosine et de la pepsine étant respectivement de 3,5 et 2,0 (HILl, NoAkes et Lowe, I970). Ainsi, in vitro, la vitesse de digestion pepsique diminue davantage pour les protéines du soja que pour celles du lait, lorsque le pH du milieu s'élève de 2 à 4 (KRATZER et PORTER, I962). Les quantités d'enzymes sécrétées dans la caillette ont probablement été réduites, comme l'ont observé Garno't et al. (I974 et r977) et Williams, Roy et Giliıes (I976) avec des protéines de lactosérum, de soja et de poisson. L'arrivée plus rapide dans le duodénum d'une quantité plus importante d'azote protéique avec nos aliments expérimentaux s'accorde avec les résultats de TERnouTh et al. (I975) et de GuiL, TEAU et al. (I975). Cela pourrait rendre plus difficile la digestion les protéines du soja et de la féverole par les protéases pancréatiques (FAUCONNEAU et MICHEL, I970), d'autant plus que la sécrétion de ces dernières est parfois diminuée lorsque les aliments d'allaitement contiennent des protéines de substitution (TERNouth et al., I975). L'ensemble de ces modifications expliquerait au moins en partie, l'utilisation digestive apparente moins élevée des matières azotées du soja et surtout de la féverole par rapport à celles du lait (respectivement 80, 67 et $95 \mathrm{p}$. I0o, d'après GUILLOTEAU et al., I977). L'influence de la vitesse d'évacuation gas- 
trique peut être appréciée en infusant plus ou moins rapidement les aliments dans le duodénum proximal; les protéines du lait et celles du poisson ont alors une utilisation digestive plus élevée ( 2 à 6 points) quand la vitesse d'infusion de l'aliment est lente (simulation de l'évacuation gastrique des protéines du lait) que lorsqu'elle est rapide (simulation du transit obtenu avec les protéines du poisson) (GUII, IOTEAU $^{-}$ et TOULIEC, travaux en cours).

L'analyse des correspondances permet de mettre en évidence certains phénomènes qui n'apparaissaient pas au préalable. Avec les aliments Soja et Féverole, l'azote non protéique quitte la caillette plus lentement que tous les autres constituants étudiés; en revanche, avec l'aliment Témoin, sa sortie est plus rapide que celles de 1'azote total et de l'azote protéique. Cela indique qu'une part importante des protéines du lait (caséine) doit être hydrolysée avant de passer dans le duodénum; il n'en est pas de même pour les protéines de substitution, qui ne coagulent pas. Avec chacun des trois aliments, les différences individuelles ne sont pas systématiques et les variations importantes observées suivant la journée de mesures (fig. 6 et 7) sont indépendantes du veau prélevé. Elles seraient surtout dues à un découpage arbitraire de la journée de mesures en sept périodes. En raison de l'effet comipensatoire de celles-ci, il semble plus logique de raisonner à partir des quantités cumulées. Ainsi, les variations dues à la journée de mesures elle-même ne portent plus que sur la première période (fig. 6). Avec les trois aliments, il y aurait deux phases d'évacuation gastrique bien distinctes. Cela semble intéressant sur le plan physiologique; toutefois il convient de se demander quelle est la part de l'effet manipulation dans la signification des différences apparues au cours de la première demi-heure de mesures. C'est pourquoi nos résultats devraient être confrontés à ceux obtenus en utilisant d'autres techniques d'étude (absorption des différents nutriments, etc.).

En conclusion, le remplacement de la majeure partie des protéines du lait par des concentrés protéiques provenant du soja et surtout de la féverole entraîne une accélération de l'évacuation gastrique des matières azotées et des matières grasses qui est probablement due à l'absence de coagulation de l'aliment. L'hyd rolyse et la dénaturation des protéines dans la caillette semblent moins prononcées, principalement dans le cas de la féverole. La digestion des aliments dans la caillette est donc influencée par l'origine des protéines alimentaires. Cela explique en partie l'utilisation digestive moins élevée des protéines de substitution.

Accepté pour publication en août 1978 .

\section{Remerciements}

A. J. Culioli et D. LE Douaron du Laboratoire de Recherches de Technologie Laitière de I'I.N.R.A. (Centre de Rennes), qui nous ont fourni le concentré protéique de féverole utilisé dans cette étude.

\section{Summary}

Utilization of proteins by the preruminant veal calf

VII. - Influence of replacing milk protein by soya bean

or field-bean proteins on gastric emptying

An experiment was carried out in the preruminant calf to study the effects on the gastric emptying of replacing skim milk protein by a soya bean or a field bean protein concentrates (Table I). The first concentrate was a soya bean oil meal treated by moist heating and cleared of its 
sucrose and a galactosides by ethanol extraction. The second one was prepared from dehulled field bean seeds by aqueous extraction at $\mathrm{pH} 8.5$. Seven preruminant calves were fitted with a duodenal reentrant cannula (Table 2). During 7 hours after the morning feed, abomasal digesta was collected. The data were treated by correspondance analysis.

When using the 2 milk substitutes containing plant proteins, the abomasal emptying of total nitrogen, protein nitrogen and lipids was accelerated (Table 3, fig. I, 3 and 5) and protein was less hydrolysed (fig. 2), mainly with field bean. The output of total nitrogen and protein nitrogen were respectively multiplied by I.2 and I.7 (soya bean) and I.6 and 2.3 (field bean). The speed of non protein nitrogen output relatively to that of protein nitrogen suggested that casein must be partly hydrolysed before leaving the abomasum; this was not necessary for the plant proteins which did not coagulate (fig. 5). The pH of the abomasal outflow was higher with the field bean diet (fig. 4). For each milk substitute, individual variations with the day of measure were large but were independant of the calf used (fig. 6 and 7 ).

Digestion in the abomasum seems to be affected by the origin of the dietary protein. The changes observed when replacing milk protein by soya bean or field bean proteins might partly account for the lower digestibility of the latter.

\section{Références bibliographiques}

Asir R. W., 1962. Gastro-intestinal reentrant canulea for studies of digestion in sheep. Anim. Prod., 4, 309-31 2.

Ash R. W., I964. Abomasal secretion and emptying in suckled calves. $J$. Physiol, 172, 425-438.

BFLL, F. R, Holbrooke S. E., Titchen D. A., I 977. A radiological study of gastric (abomasal) emptying in calves before and after vagotomy. J. Physiol., 272, 48 I-493.

BEI, F F. R., RAzIG S. A. D., I973a. Gastric emptying and secretion in the milk fed calf. $J$. Physiol., 288, 499-5Iz.

BFLL, F. R., RAZIG S. A. D., I $973 b$. The effect of some molecules and ions on gastric function in the milk-fed calf. $J$. Physiol., 228, 513-526.

BeNZECRI J. P. et 23 collaborateurs, 1973. L'analyse des données. Tome II, 6ro p., Dunod, Paris.

Colvin B. M., Lowe R. A., RAMsey H. A., I 969 . Passage of digesta from the abomasum of calf fed soy flour milk replacers and whole milk. J. Dairy Sci., 52, 687-688.

FaUCONnEAU G., Micheid, M. C., 1970. The role of the gastro-intestinal tract in the regulation of protein metabolism. In MUNRo H. N. Mammalian protein metabolism, 4, $4^{81-522}$, Ac. Press. New-York.

likantzen J. F., Toullec R., Thivend P., Mathiev C.-M., i973. Influence de la coagulation des protéines sur la vidange stomacale chez le veau préruminant. Ann. Biol. anim. Bioch. Biophys., 13, 718-72I.

Gaknot P., Valles E., Thapon J. L., Touli,e R., Tomassone R., Ribadeat-Dumas B., i 974. Influence of dictary proteins on rennin and pepsin content of preruminant calf vell. $J$. Dairy Res., 41, 19-23.

Garnot P., Tovliec R., Thapon J. L., Martin P., Minh-Thu-Hoang, Mathiec C.-MI., RibadeatDumas B., 1977. Influence of age, dietary protein and weaning on calf abomasal enzymatic secretion. J. Dairy Res., 44, 9-23.

Guiliotead P., LE Calve J. L., 1977. Technique de réalisation d'une poche abomasale chez le veau en vue de l'obtention de suc gastrique pur. Ann. Biol. anim. Bioch. Biophys., 17, I047ro6o.

Guililoteau P., Paruelle J.-L., Toullec R., Mathieu C.-M., 1975. Utilisation des protéines par le veau préruminant. III. Influence du remplacement des protéines du lait par celles du poisson sur la vidange stomacale. Ann. Zootech., 24, 243-253.

Guilioteat P., TOuli,ec R., Culiol, J., LE Douaron D., I977. Utilisation des protéines par le veau préruminant à l'engrais. V. Utilisation digestive des protéines du poisson, du soja et de la féverole. Ann. Zootech., 26, I 5-28.

Hill K. J., NOAkes D. E., Lowe R. A., 197o. Gastric digestive physiology of the calf and piglet. In Phillipson A. T., Physiology of digestion and metabolism in the ruminant, I66-I 79, Oriel Press, Newcastle upon Tyne.

Kratzer F. H., Porter J. W. G., I962. The effect of $\mathrm{pH}$ on the digestion of protein in vitro by pepsin. Br. J. Nutr., 16, 579-584.

LFIBHOLZ J., I975. Dietary effects on the flow of nutrients from the abomasum of the preruminant calf. Aust. J. Agric. Res., 26, 623-633. 
Mathieu C.-M., ig68. Fitude de la vidange stomacale du lait entier chez le veau préruminant. Ann. Biol. anim. Bioch. Biophys., 8, 581-583.

MYLREA P. J., I966a. Digestion of milk in young calves. I. Flow and activity of the contents of the small intestine. Res. Vet. Sci., 7, 333-34I.

MYLREA P. J., 1966b. Digestion of milk in young calves. II. The absorption of nutrients from the sniall intestine. Res. Vet. Sci., 7, 394-406.

RAO C. R., I952. Advanced statistical methods in biometric research, 390 p., J. WILEY, New-York.

Roy J. H. B., Stobo I. J. F., I975. Nutrition of the preruminant calf. In Me DoxaLD J. W., WARNER A. C. I. Digestion and metabolism in the ruminant. Proc. IV th int. Symp. on ruminant physiology, 30-48. Univ. of New-England, Publ. Unit., Armidale.

Smith R. H., I959. The development and function of the rumen in milk fed calves. J. A gric. Sci, 52, $72-78$.

Smith R. H., Sissons J. W., 1975. The effect of different feeds, including soya-bean products, on the passage of digesta from the abomasum of the preruminant calf. $B r . J . N u t r ., 33,329-349$.

Sissons J. W., Smith R. H., I975. Field bean (Vicia faba L) protein in feeds for preruminant calves. Proc. Nutr. Soc., 34, I02A.

Ternouth J. H., Roy J. H. B., Thompson S. Y., ToOThil, J., Gil.tes C. M., EDWARds-WebB J. D., r975. Concurrent studies of the flow of digesta in the duodenum and of exocrine pancreatic secretion of calves. 3. Further studies on the addition of fat to skim milk and the use of non-milk proteins in milk-substitute diets. Br. J. Nutr., 33, I8 1-196.

Toulla, R., Thivend P., Mathieu C.-M., I97I. Utilisation des protéines du lactosérum par le veau préruminant. I. Vidange stomacale comparée du lait entier et de deux laits de remplacement ne contenant que des protéines de lactosérum comme source de matières azotées. Ann. Biol. anim. Bioch. Biophys., 11, 435-453.

Williams V. J., Roy J. H. B., Gili.ies C.-M., I976. Milk-substitute diet composition and abonasal secretion in the calf. Br. $J$. Nutr., 36, 3I 7-335. 